\title{
Neural changes associated with appetite information processing in schizophrenic patients after 16 weeks of olanzapine treatment
}

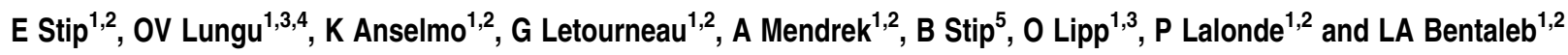

There is evidence that some atypical antipsychotics, including olanzapine, can produce unwanted metabolic side effects, weight gain and diabetes. However, neuronal correlates of change related to food information processing have not been investigated with these medications. We studied the effect of a pharmacological manipulation with an antipsychotic known to cause weight gain on metabolites, cognitive tasks and neural correlates related to food regulation. We used functional magnetic resonance imaging in conjunction with a task requiring visual processing of appetitive stimuli in schizophrenic patients and healthy controls before and after 16 weeks of antipsychotic medication with olanzapine. In patients, the psychological and neuronal changes associated following the treatment correlated with appetite control measures and metabolite levels in fasting blood samples. After 16 weeks of olanzapine treatment, the patients gained weight, increased their waist circumference, had fewer positive schizophrenia symptoms, a reduced ghrelin plasma concentration and an increased concentration of triglycerides, insulin and leptin. In premotor area, somatosensory cortices as well as bilaterally in the fusiform gyri, the olanzapine treatment increased the neural activity related to appetitive information in schizophrenic patients to similar levels relative to healthy individuals. However, a higher increase in sensitivity to appetitive stimuli after the treatment was observed in insular cortices, amygdala and cerebellum in schizophrenic patients as compared with healthy controls. Furthermore, these changes in neuronal activity correlated with changes in some metabolites and cognitive measurements related to appetite regulation.

Translational Psychiatry (2012) 2, e128; doi:10.1038/tp.2012.53; published online 19 June 2012

\section{Introduction}

There is increasing evidence that some atypical antipsychotics, including olanzapine, can produce metabolic side effects. In a recent review, De Hert et al. ${ }^{1}$ concluded that 'although some drug receptor-binding affinities correlate with specific cardiovascular and metabolic abnormalities, the exact pharmacological mechanisms underlying these associations remain unclear'. In fact, a recent meta-analysis confirmed that olanzapine treatment resulted in more weight gain, cholesterol and glucose elevation than all other secondgeneration antipsychotics, except for clozapine. $^{2}$ For instance, it has been shown that olanzapine produced statistically more weight gain than risperidone (mean difference: $2.44 \mathrm{~kg}$ ), aripiprazole and ziprasidone (mean difference: 3.9 and $3.82 \mathrm{~kg}$, respectively). Naturalistic and controlled clinical trials ${ }^{3-7}$ suggest that one of the ways olanzapine can lead to changes in the patterns of food intake is by increasing appetite $^{8-12}$ preference for certain diet. ${ }^{13,14}$

Moreover, it has been suggested that antipsychotics in general can contribute to decreased caloric dissipation via diminished physical activity, ${ }^{6}$ due to their sedative effects. ${ }^{15}$ However, Gothelf and co-workers ${ }^{13}$ prospectively measured body mass indices, caloric intake and energy expenditure in 10 olanzapine-treated subjects and in a matched group of patients treated with haloperidol, and found that at 4-week follow-up, patients had increases in both caloric intake and body mass index, without any change in their resting and daily energy expenditure. Thus, caloric intake seems to play an important role in olanzapine-induced weight gain. Indeed, one study found that $22.2 \%$ of the female patients and $6.4 \%$ of the males fulfilled the criteria for binge eating disorder when both clozapine and olanzapine were taken together. ${ }^{16}$

As this brief survey of the literature illustrates, long-term treatment with olanzapine in schizophrenic patients could lead to significant weight gain and its associated health problems, such as diabetes and heart disease. With the increased prevalence of metabolic illness in schizophrenia, it is of great clinical importance to determine to what extent the olanzapine induces changes that reflect trait and state eating behaviors. The deleterious effects of olanzapine on serum lipids can be independent of the body mass index or, in addition, to weightrelated effects. ${ }^{17}$ Albaugh et al. ${ }^{18}$ recently proposed an interesting mechanism to explain why olanzapine might cause metabolic side effects leading to obesity and diabetes based

\footnotetext{
${ }^{1}$ Department of Psychiatry, Université de Montréal, Montréal, QC, Canada; ${ }^{2} \mathrm{CHUM}$, Centre de Recherche Fernand Seguin, Université de Montréal, Notre Dame Hospital, Montréal, QC, Canada; ${ }^{3}$ Centre de Recherche de l'Institut Universitaire de gériatrie de Montréal, Université de Montréal, Montréal, QC, Canada; ${ }^{4}$ Department of Research, Donald Berman Maimonides Geriatric Center, Montréal, QC, Canada and ${ }^{5}$ École Polytechnique de Montréal, Montréal, QC, Canada

Correspondence: Dr E Stip, CHUM, Centre de Recherche Fernand Seguin, Université de Montréal, Notre Dame Hospital, 2065, Alexandre de Seve, Montreal, QC, H2L 2W5 Canada.

E-mail: emmanuel.stip@ umontreal.ca
}

Keywords: appetite; fMRI; insula; metabolic changes; olanzapine; schizophrenia

Received 27 Feburary 2012; revised 30 April 2012; accepted 6 May 2012 
on an animal model in male Sprague-Dawley rats. They found a tendency for increased ${ }^{14} \mathrm{C}$-2-deoxyglucose uptake into fat depots of fed rats and a free fatty acid uptake into fat depots elevation, in parallel with increased adipose tissue lipogenesis. These results, consistent with the olanzapine-induced hyperglycemia hypothesis, suggest that this drug exerts direct metabolic effects that together favor increased accumulation of fuel into adipose tissue, yet they do not explain completely the complexity of the side effects found in humans, such as the cognitive aspect relative to food intake or the change in eating behavior in psychotic patients treated with olanzapine. To date, the associations between behavioral phenotypes and genetic variability in psychotropic medication responses, including the effects on obesity, are still unknown. For instance, both the motivational salience to eat and the factors involved in eating termination as a function of the hedonic value of food are all insufficiently investigated. However, there is increasing evidence ${ }^{19-21}$ to suggest that there are links between obesity, perception of appetitive cues, food intake and reward, which are mediated by specific changes in the brain activity. Thus, it may be the case that such cognitive processes may account, at least in part, for the individual variability in changes of food intake and body weight associated with antipsychotic treatment in schizophrenia.

In a previous study, we showed that metabolic and cognitive correlates of dysregulated appetite in schizophrenia may be related to common neural substrates. ${ }^{22}$ However, in that study, patients were prescribed several different antipsychotic medications. This study was designed to examine changes in the brain activity associated with 16 weeks of olanzapine treatment in schizophrenic patients. Thus, the studied population in this study is pharmacologically homogeneous. In addition, we were interested to conduct exploratory analyses that reveal the correlations between psychological and neuronal changes and appetite control measures and metabolite levels in fasting blood samples.

\section{Materials and methods}

Participants. A cohort of 24 consecutive outpatients (19 men and 5 women) suffering from schizophrenia according to the DSM-IV criteria, who were not previously exposed to olanzapine and who were placed under olanzapine treatment by their psychiatrist, were initially recruited for participation in this study (Table 1). All patients, with the exception of three who were under no medication, switched from other antipsychotics to olanzapine as a result of an insufficient clinical response. The switch was done without a washout period as follows: 12 from risperidone, 6 quetiapine, 2 haloperidol, 1 clozapine and 1 perphenazine. The study was reviewed and approved by the Institutional Review Board of Centre de Recherche Fernand Seguin and by the Research Ethics Committee of the Centre de Recherche of the Institut Universitaire de Gériatrie de Montréal, and all the participants gave their voluntary and informed consent. There was no subject with a diabetes mellitus or with abnormal glucose level at baseline.

During the trial, dosing of olanzapine was flexible depending of the clinical status of the patients with a daily mean dosage of $16.18 \mathrm{mg}$ (s.d. 6.97). However, of all 24 patients recruited initially, only 15 completed both the 16 weeks of olanzapine treatment and the second scanning session. The other 9 patients either interrupted their treatment (2 participants) or did not wish to participate in the second imaging session (7 participants). Thus, only data from these 15 patients are reported here. In addition, 11 healthy individuals were scanned only once using the same experimental protocol and served as controls. Only the data from 10 of them was used in the study as one healthy individual displayed unusually large ventricles, thus posing a challenge for the anatomical standardization of his brain images. Table 1 summarizes the demographic characteristics of all participants, both patients and controls.

Task description and experimental design. All participants, healthy controls and patients with schizophrenia alike, underwent whole-brain functional magnetic resonance imaging (fMRI) at high field (3 Tesla). Each participant was in a controlled satiated state. In each fMRI session, subjects participated in a passive viewing task during two experimental conditions: Appetizing (AC) and Neutral (NC). In all, 10 blocks of 11 pictures each were presented in total, with 5 blocks in the NC condition (emotionally neutral pictures such as a tourist, rocks, and so on) and 5 blocks in AC condition (images of food pictures: cakes, pizza, and so on). The two categories of pictures were selected from the IAPS (International Affective Picture System) ${ }^{23}$ and were matched as much as possible in terms of visual complexity, while being rated for their appetizing appeal. The selection of the images was carried out as follows: (a) appetite-related and appetite-unrelated images were selected from the IAPS database by the experimenters; these images had similar valence and intensity ratings, based on the normative data from the IAPS database; (b) the selected images were

Table 1 Demographic characteristics of schizophrenic (SZ) patients and healthy controls

\begin{tabular}{lccc}
\hline & $\begin{array}{c}\text { Healthy controls who } \\
\text { participated in the study }\end{array}$ & $\begin{array}{c}\text { All SZ recruited initially } \\
\text { in the study }\end{array}$ & $\begin{array}{c}\text { All SZ who followed the treatment } \\
\text { and completed the 2 scanning sessions }\end{array}$ \\
\hline Total number & 10 & 24 & 15 \\
Females/males & $2 / 8$ & $5 / 19$ & $4 / 11$ \\
Age (years; $m \pm$ s.d.) & $33.9(10.76)$ & $30.04(9.41)$ & $35.83(10.12)$ \\
Race/ethnicity & $100 \%$ Caucasians & $91.66 \%$ Caucasians vs & $86.66 \%$ Caucasians \\
& & $8.33 \%$ Caribbean & vs $13.33 \%$ Caribbean
\end{tabular}

Note: One healthy control (male, 34 years old) was excluded from the study (his demographic data are not presented in this table) because of unusually large ventricles, which posed problems for the standardization of the anatomical data. 
mixed-up and then subsequently given to a group of healthy controls and schizophrenic patients (other than the ones involved in this study) who classified the images into appetite-related and appetite-unrelated (neutral); (c) only the images who were similarly classified by both the experimenters and these participants and which had similar valence and intensity based on the IAPS normative ratings were included in the study.

Each block lasted for $31.68 \mathrm{~s}$ and was separated from others by resting periods of $14.4 \mathrm{~s}$, during which subjects watched a cyan screen. This passive viewing task was used to avoid recruiting other cognitive processes in conjunction with the presentation of the stimuli. Given that appetite is a state that may last for some time after food stimuli are presented, the neutral images were always presented before the appetitive ones (Figure 1a), ensuring that appetite was not provoked during the NC condition. The subjective responses to food images were assessed at the end by asking subjects to rate the average intensity of the appetite felt during the session, on a visual analog rating scale ranging from 0 (not appetizing at all) to 5 (biggest appetite ever had in one's lifetime). For patients, the first magnetic resonance examination was scheduled during the first week of the study, before taking olanzapine medication, and was repeated at the end of the follow-up, at week 16. All the participants were scanned approximately at the same time during both scans as hunger level and appetite changes over time of day.

Clinical assessment and physiological measures. Biometrical data, such as body weight, body mass index, abdominal circumference and blood pressure, were collected at weeks 1, 2 and 16. At the same times, blood samples were taken, and analyzed for fasting glucose, insulin, prolactin, leptin, ghrelin and lipid profiles. Clinical follow-up also took place at weeks 1, 2, 8, 12 and 16. Each time, patients were assessed using the Positive and Negative Symptoms Scale $^{24}$ and Calgary Depression Scale for Schizophrenia ${ }^{25}$ by a single trained physician. Patients completed the ThreeFactor Eating Questionnaire (TFEQ) ${ }^{26}$ at weeks 1 and 16 to assess changes in their appetite and eating-related behavior. The same procedure, but no treatment, was employed for the group of healthy individuals (control group). In these participants, the eating habits were assessed with the TFEQ and their weight and body size were measured. The blood samples were indeed collected after an overnight fast. The $3 \mathrm{~h}$ was used as a standard period of time for all participants elapsed between the last meal and the imaging session.

Analysis of psychological and physiological data. The changes in psychological and physiological variables from baseline to 16 weeks after treatment pertaining to patients were analyzed using Wilcoxon's rank-sum test, a nonparametric version of the paired sample $t$-test, which does not make any assumption regarding the normal distribution of the data. Given that we had a priori hypotheses about the direction in which these variable would change (for example, an increase in weight), we used one-tail significance threshold $P<0.05$. The correlations between psychological, physiological and neuroimaging data were performed using both Pearson's correlation coefficient and Spearman's rank correlation coefficient (which is less sensitive to outlier values), and only when the two coefficients agreed did we reporte the correlation as being significant.

Imaging parameters. A 3Tesla whole body MR system (Magnetom Trio, Siemens Medical Systems, Erlangen, Germany) was used for image acquisition. In each fMRI session, structural images were acquired in sagittal plane by using an MPRAGE (magnetization-prepared rapid acquisition) imaging sequence (176 slices, $\mathrm{TR}=13 \mathrm{~ms}$; $\mathrm{TE}=$ $4.92 \mathrm{~ms} ; \mathrm{FA}=25^{\circ} ; \mathrm{FoV}=256 \mathrm{~mm}^{2}$; matrix size $=256 \times 256$; slice thickness $=1 \mathrm{~mm}$, voxel size $=1 \mathrm{~mm}^{3}$ ). Whole brain $\mathrm{fMRI}$ was performed using an echo-planar imaging sequence measuring blood oxygenation level-dependent (BOLD) signal $\left(\mathrm{TR}=3000 \mathrm{~ms} ; \mathrm{TE}=30 \mathrm{~ms} ; \mathrm{FA}=90^{\circ} ; \quad \mathrm{FoV}=224 \mathrm{~mm}^{2}\right.$; matrix size $=64 \times 64$; slice thickness $=3.5 \mathrm{~mm}$, voxel size $=3.5 \mathrm{~mm}^{3} ; 41$ slices, 160 volumes). Functional slices were oriented in transverse plane and were angled to be parallel to the AC-PC line. An inline retrospective motion correction algorithm was employed while the echo-planar imaging images were acquired.

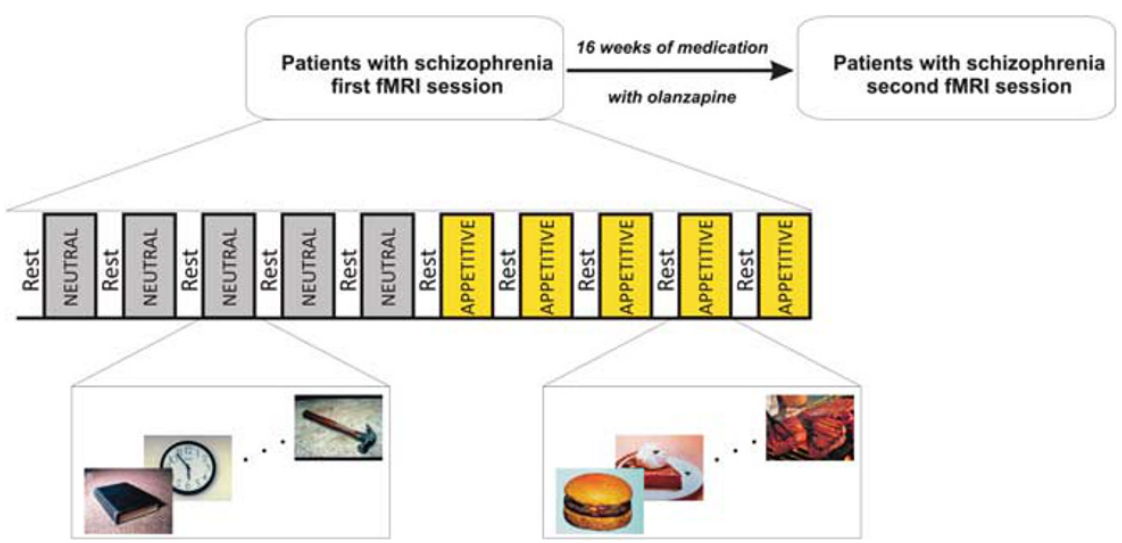

Figure 1 Patients were scanned before and after 16 weeks of olanzapine treatment. In each functional magnetic resonance imaging (fMRI) session, given that appetite is a state that may last for some time after food stimuli are presented, the blocks of appetitive stimuli were always presented last to prevent the appetite to develop during the presentation of the neutral stimuli. In each block, there were 11 neutral or appetite-related pictures (depending on the block type) presented. 
Preprocessing of imaging data. The Brain Voyager QX (Brain Innovation B.V., Maastricht, The Netherlands) software was used for fMRI data preprocessing and analysis. The functional bi-dimensional images were preprocessed to correct for the difference in time slice acquisition. In addition to linear de-trending, a high-pass filter of three cycles per time course (frequency domain) was applied to the corrected twodimensional slices. Thereafter, the functional series was preprocessed to correct for possible motion artifacts and to ensure that movements in any direction did not exceed $3 \mathrm{~mm}$. These images were subsequently used to reconstruct the three-dimensional functional volume for each subject and run. The three-dimensional functional volume was aligned with the corresponding anatomical volume, and both were normalized to standard Talairach space. ${ }^{27}$ Spatial smoothing using a Gaussian kernel at $8 \mathrm{~mm}$ full-width at half-maximum was applied to the three-dimensional functional data.

Analysis of imaging data. A blocked-design approach was employed for data analysis. For each scanning session, we defined two predictors (Appetitive and Neutral), corresponding to the blocks of presentation of appetitive and neutral images. These predictors were first entered as fixed factors in a single subject general linear model, and then the parameters of this general linear model were subsequently entered into a second level of analysis corresponding to a random-effect general linear model that was used for group analysis. ${ }^{28}$ The criteria used to display the activation maps were a cluster size of 108 adjacent significant voxels (corresponding to a volume of $108 \mathrm{~mm}^{3}$ or four functional voxels of $3 \times 3 \times 3 \mathrm{~mm}^{3}$ each) and a statistical threshold for each voxel in the cluster of $P<0.005$ (uncorrected), which in turn corresponds to $P<0.05$ family-wise error for a maximum number of 10 clusters of activation surpassing this 0.005 threshold.

We were interested in one interaction contrast in our main analysis: the difference between sessions (Session $2>$ Ses- sion 1) in schizophrenic patients regarding the difference between the two types of stimuli (AC > and NC). This contrast (assessed in each voxel using a one-sample $t$-test for the variable describing the difference (Appetitive ${ }_{\text {Session }} 2^{-}$ Neutral $_{\text {Session 2 }}$-(Appetitive Session 1 $_{1}-$ Neutral $\left._{\text {Session 1 }}\right)$ ) identified the brain regions, which became more or less sensitive to appetitive stimuli as a result of olanzapine treatment. In addition, the activated clusters obtained as a result of this contrast were defined as regions of interest (ROIs) and further analyses were performed separately for each of them by taking into account the estimation of the BOLD signal change averaged over all voxels in the ROI and using the SPSS software (SPSS, Chicago, IL, USA). Specifically, we performed correlation between the changes in the brain activity in these ROls and various psycho-physiological measures (for example, TFEQ or Positive and Negative Symptoms Scale scores, concentration of metabolites, and so on). In addition, we estimated the values of the BOLD signal change in these ROIs for the control subjects to identify in which of them the neuronal activity was: (a) perturbed before the treatment (relative to controls) and brought back to 'normal' by the treatment or (b) at 'normal' levels before the treatment and then perturbed as a result of the clinical intervention. We also performed a secondary analysis, similar to that in our previous paper, ${ }^{22}$ where we compared the brain activity specific to appetitive stimuli among patients after the treatment with that of healthy controls. Whereas previously we compared healthy controls and schizophrenic patients under various antipsychotic medications (except olanzapine), here we assessed these group differences after patients were all under the influence of the same antipsychotic, namely olanzapine.

\section{Results}

Physiological changes following olanzapine treatment. At the end of 16 weeks of olanzapine treatment, $73 \%$ of the patients (11/15) gained between 2 and $7 \mathrm{~kg}$ in weight, a

Table 2 Changes in SZ patients before and after the treatment

\begin{tabular}{|c|c|c|c|}
\hline & $S Z$ before treatment $(m \pm$ s.d.) & $S Z$ after treatment $(m \pm s . d)$. & $\begin{array}{c}\text { One-tailed } \\
\text { P-value }\end{array}$ \\
\hline $\begin{array}{l}\text { Weight (kg) } \\
\text { TFEQ }\end{array}$ & $82.00 \pm 16.76$ & $83.52 \pm 15.93$ & 0.03 \\
\hline $\begin{array}{l}\text { Factor } 1 \\
\text { Factor } 2 \\
\text { Factor } 3\end{array}$ & $\begin{array}{l}8.60 \pm 3.83 \\
5.46 \pm 2.69 \\
4.06 \pm 2.84\end{array}$ & $\begin{array}{l}8.40 \pm 3.83 \\
5.46 \pm 2.85 \\
4.13 \pm 3.04\end{array}$ & $\begin{array}{l}0.32 \\
0.27 \\
0.27\end{array}$ \\
\hline $\begin{array}{l}\text { PANSS, positive } \\
\text { PANSS, negative } \\
\text { PANSS, general } \\
\text { PANSS, total } \\
\text { Glucose }\left(\mathrm{mmol}^{-1}\right) \\
\text { Cholesterol }\left(\mathrm{mmolI}^{-1}\right) \\
\text { Triglyceride }\left(\mathrm{mmol}^{-1}\right) \\
\text { HDL }\left(\mathrm{mmol} \mathrm{I}^{-1}\right) \\
\text { LDL }\left(\mathrm{mmol} \mathrm{I}^{-1}\right) \\
\text { Chol LDL } \\
\text { Insulin }\left(\mathrm{pmolI} \mathrm{I}^{-1}\right) \\
\text { Prolactin }\left(\mu \mathrm{I}^{-1}\right) \\
\text { Leptin }\left(\mathrm{ng} \mathrm{ml}^{-1}\right) \\
\text { Ghrelin }\left(\mathrm{pg} \mathrm{ml}^{-1}\right)\end{array}$ & $\begin{array}{c}\mathbf{2 0 . 8 0} \pm \mathbf{5 . 7 9} \\
18.06 \pm 4.21 \\
39.26 \pm 9.36 \\
78.13 \pm 15.05 \\
4.90 \pm 0.49 \\
4.31 \pm 1.09 \\
\mathbf{1 . 1 0} \pm \mathbf{0 . 7 4} \\
1.16 \pm 0.24 \\
2.65 \pm 0.97 \\
3.98 \pm 1.62 \\
\mathbf{4 5 . 0 6} \pm \mathbf{3 0 . 4 5} \\
27.98 \pm 51.13 \\
\mathbf{9 . 1 7} \pm \mathbf{1 4 . 0 8} \\
\mathbf{1 0 6 2 . 4 6} \pm \mathbf{3 7 9 . 3 3}\end{array}$ & $\begin{array}{c}\mathbf{1 8 . 5 3} \pm \mathbf{4 . 9 5} \\
18.53 \pm 5.05 \\
36.53 \pm 10.93 \\
73.60 \pm 18.93 \\
5.05 \pm 1.02 \\
4.45 \pm 0.54 \\
\mathbf{1 . 3 7} \pm \mathbf{0 . 8 8} \\
1.17 \pm 0.34 \\
2.65 \pm 0.54 \\
4.00 \pm 1.22 \\
\mathbf{7 4 . 7 6} \pm \mathbf{4 9 . 7 2} \\
16.12 \pm 10.33 \\
\mathbf{1 1 . 7 1} \pm \mathbf{1 2 . 9} \\
\mathbf{9 8 9 . 0 9} \pm \mathbf{3 8 0 . 8 5}\end{array}$ & $\begin{array}{l}\mathbf{0 . 0 3} \\
0.47 \\
0.16 \\
0.19 \\
0.07 \\
0.21 \\
\mathbf{0 . 0 3} \\
0.47 \\
0.41 \\
0.28 \\
\mathbf{0 . 0 0 8} \\
0.45 \\
\mathbf{0 . 0 2} \\
\mathbf{0 . 0 4}\end{array}$ \\
\hline
\end{tabular}

Abbreviations: HDL, high-density lipoprotein; LDL, low-density lipoprotein; PANSS, Positive and Negative Symptoms Scale; SZ, schizophrenic; TFEQ, Three-Factor Eating Questionnaire.

Bold indicates significant differences pre- to post-treatment $(P<0.05$, one-tailed), measured with Wilcoxon's signed-rank test. 
change that was statistically significant (Table 2). The treatment also significantly reduced Positive and Negative Symptoms Scale positive symptoms and the plasma concentration of ghrelin, while increasing the concentration levels of triglycerides, insulin and leptin hormones.

Change in brain activity related to cognitive processing of food stimuli after olanzapine treatment. The difference in the brain activity between $A C$ and $N C$ was elevated in patients in eight brain areas, as a result of olanzapine treatment (Table 3 and Figure 2). No other brain region displayed the opposite pattern of activity: a decrease in the difference between the two experimental conditions from Session 1 to Session 2. These eight regions were located in right premotor area, fusiform (2 ROIs) and left parahippocampal (amygdala) and postcentral gyr (somatosensory cortex), as well as in right insula (2 ROls) and semi-lunar cerebellar lobule. The detailed ROI analysis revealed that in four of these regions (right premotor area, both right and left fusiform gyri and the left somatosensory cortex), olanzapine treatment brought the BOLD signal in response to appetitive stimuli in schizophrenic patients back to levels comparable with those seen in healthy controls (Figure 3a).

In the remaining regions, in both insular ROls $(\mathrm{F}(2,37)=5.72, P<0.01$ and $\mathrm{F}(2,37)=4.00, P<0.03)$, amyg dala $(\mathrm{F}(2,37)=2.98, \quad P<0.05) \quad$ and $\quad$ cerebellum $(\mathrm{F}(2,37)=4.66, P<0.02)$, the effect of the treatment was an 'overshoot' in the BOLD signal in response to appetitive images, significantly above levels seen in healthy controls (Figure 3b).

Correlations between physiological measurements and appetite regulation (TFEQ) as a result of olanzapine treatment. We observed significant negative correlations between the difference pre- and post-treatment measured for Factor 1 of the TFEQ (dietary restraint) and the same difference measured for the levels of cholesterol $(r=-0.664$; $P<0.05, N=15)$, low-density lipoprotein (LDL) $(r=-0.661$; $P<0.05, N=15)$, chol/low-density lipoprotein $(r=-0.573$; $P<0.05, N=15)$ and leptin $(r=-0.496 ; P<0.05, N=15)$. These correlations indicate that the higher the score difference in dietary restraint as a result of the treatment, the lower the difference in concentration of these metabolites and hormones in the fasting blood. For the differences pre- to post-treatment for Factors 2 (disinhibition) and 3 (hunger) of the TFEQ, we observed only positive correlations with ghrelin concentration after the treatment $(r=+0.530 ; P<0.05$, $N=15)$ and difference pre- to post-treatment in chol/LDL ( $r=+0.571 ; \quad P<0.05, \quad N=15) \quad$ blood concentrations, respectively. These show that after the treatment increased levels in ghrelin concentration are associated with poor

Table 3 Brain regions in which the difference in BOLD signal between appetitive and neutral condition increased significantly among patients as a result of olanzapine treatment

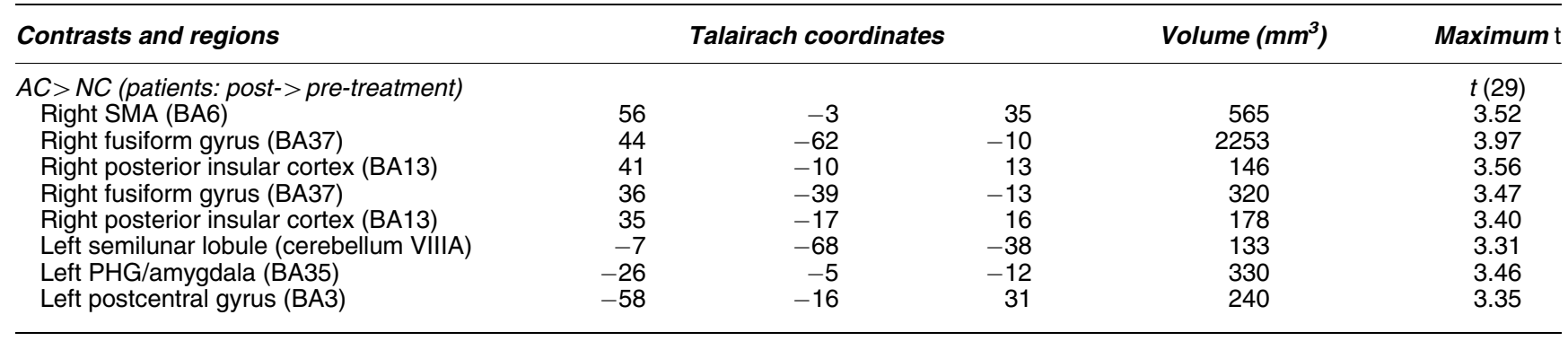

Abbreviations: AC, appetitive condition; BA, Brodmann area; BOLD, blood oxygenation level dependent; NC, neutral condition; PHG, parahippocampal gyrus; SMA, supplementary motor area.

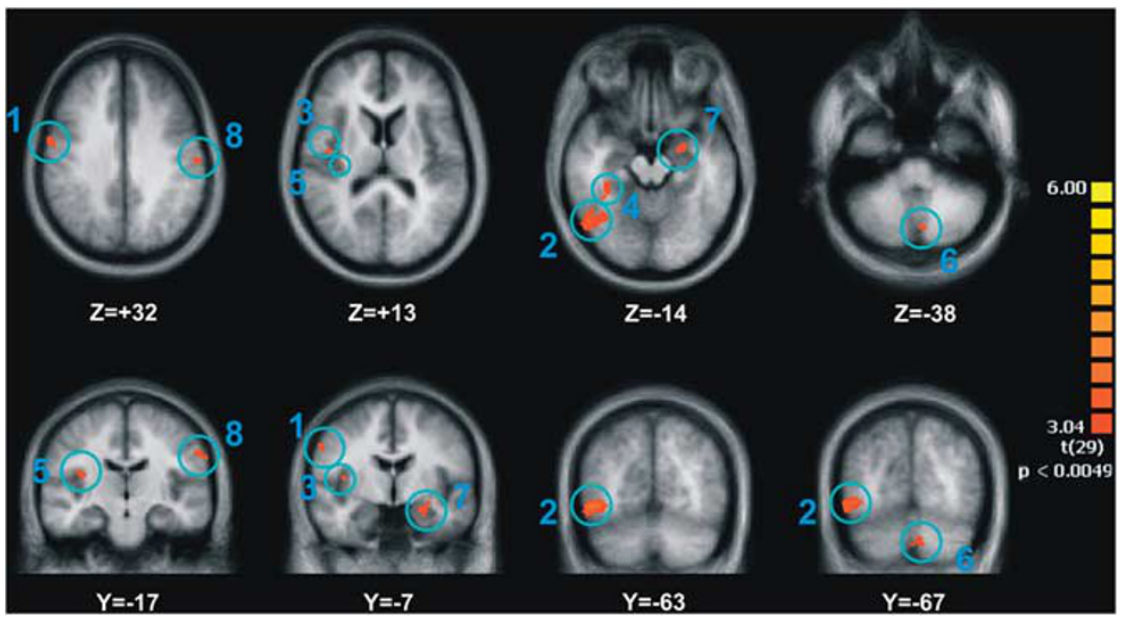

Figure 2 Brain regions in which the difference in blood oxygenation level dependent (BOLD) signal between appetitive and neutral condition increased significantly among patients as a result of olanzapine treatment. 

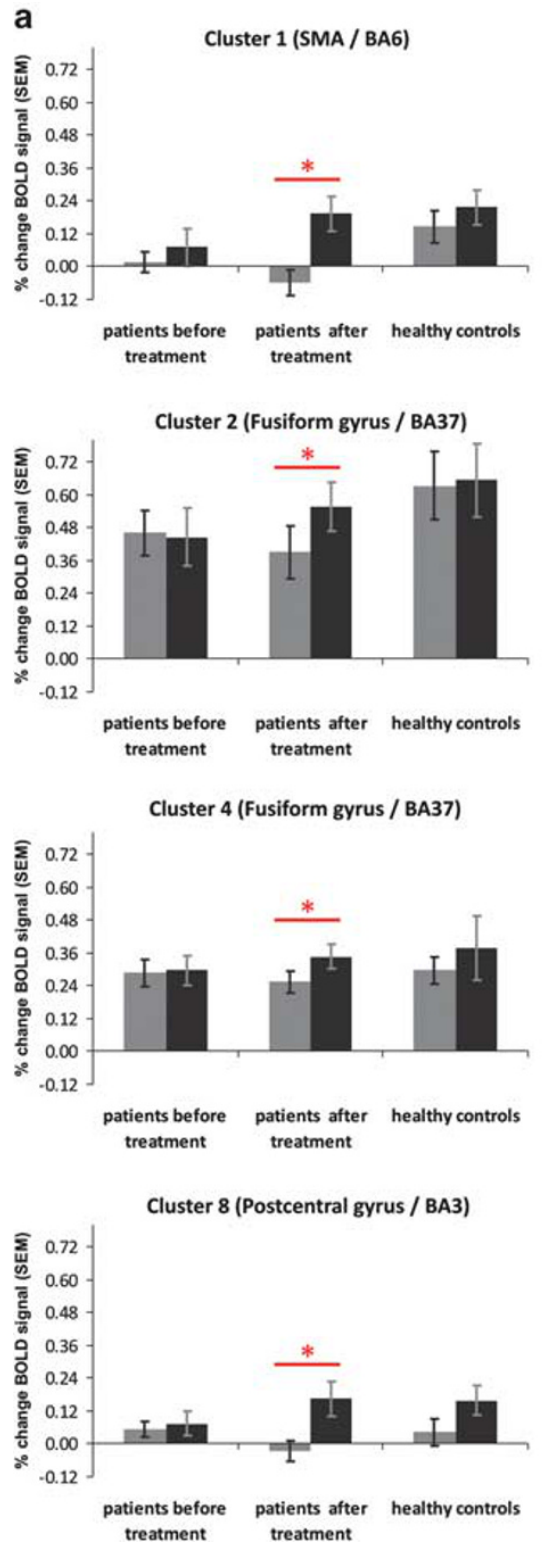

Figure 3 Changes in blood oxygenation level dependent (BOLD) signal for appetitive and neutral condition in each group (patients pre-treatment, patients post-treatment, healthy controls) in each region of interest (ROI). (a) Regions in which the BOLD signal for appetitive stimuli was similar in magnitude among patients post-treatment with that corresponding for healthy controls. (b) Regions in which the BOLD signal for appetitive stimuli was significantly higher in patients after treatment than that among healthy controls. ${ }^{*} P<0.05$.

control of eating behavior when subjects are faced with appetitive stimuli, whereas higher differences in concentration of low-density lipoproteins (chol/LDL) are associated with a high susceptibility to hunger cues. All these results indicate that the metabolic side effects of olanzapine treatment are accompanied by cognitive changes in appetite regulation and control.

\section{Correlations between physiological measurements and} BOLD signal changes as the result of olanzapine treatment. We found several correlations between changes in BOLD signal as a result of treatment, indicating an increase in sensitivity to appetitive information, and changes in physiological measurement related to
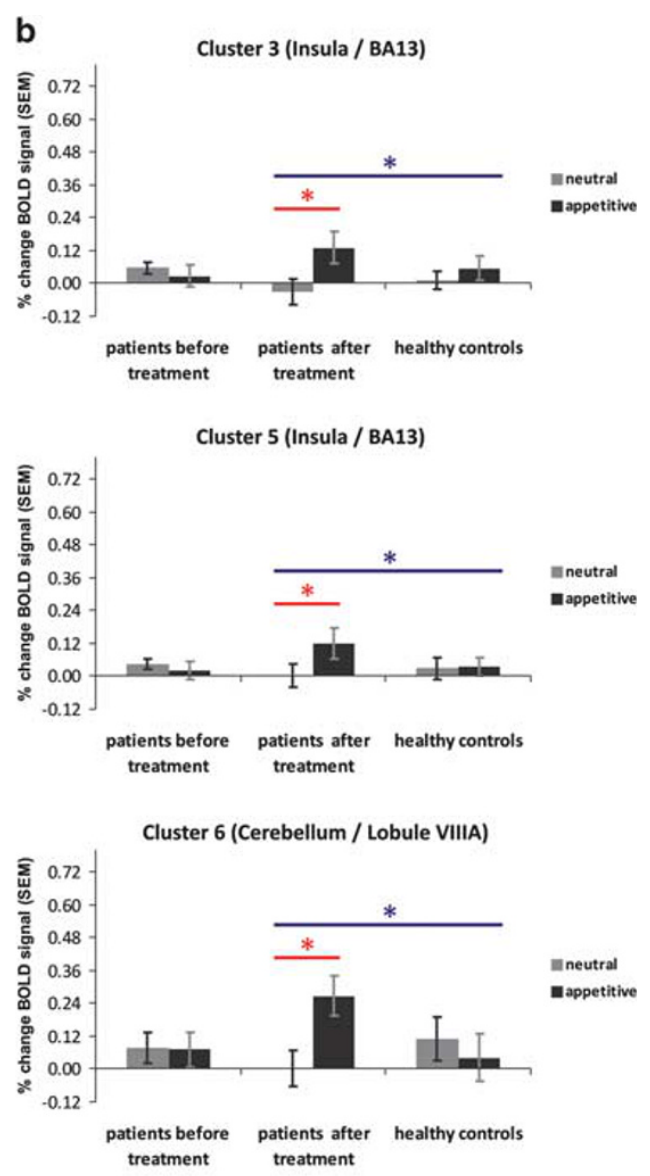

Cluster 7 (Parahippocampal gyrus / Amygdala)

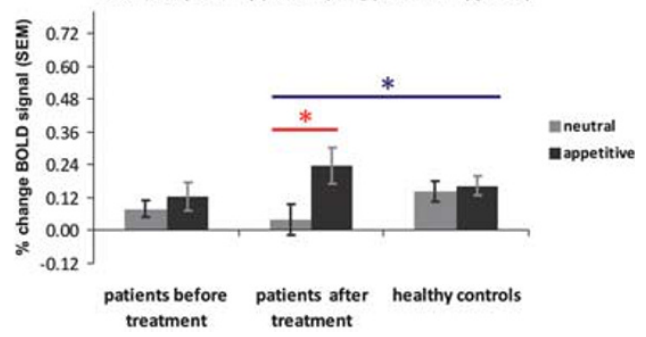

olanzapine treatment. With two exceptions, most of these correlations were negative. For example, we found that the difference in neuronal activity pertaining to appetitive stimuli in premotor area correlated negatively with that of triglycerides $(r=-0.463 ; \quad P<0.05, \quad N=15)$, in fusiform gyrus, with glucose $(r=-0.573 ; P<0.05, N=15)$ and leptin $(r=-0.519 ; P<0.05, N=15)$ concentrations, in insula, with the changes in triglyceride $(r=-0.503 ; P<0.05, N=15)$ and chol/LDL ( $r=-0.472 ; P<0.05, N=15)$ concentrations, and in amygdala, with changes in the levels of glucose $(r=-0.449$; $P<0.05, N=15)$, insulin $(r=-0.544 ; P<0.05, N=15)$ and prolactin ( $r=-0.456 ; P<0.05, N=15)$. Only in the fusiform gyrus (cluster 4 in Table 3 ), we found positive correlations between the BOLD signal and changes in high-density 
lipoprotein $(r=+0.473 ; \quad P<0.05, \quad N=15)$ and ghrelin concentrations $\quad(r=+0.679 ; \quad P<0.05, \quad N=15)$. It is interesting to note that of all regions, the activity in the fusiform gyrus, whose level is brought back to 'normal' after olanzapine treatment, was the one that correlated with changes in many metabolites, indicating perturbations of appetite (that is, positive correlation with ghrelin and negative correlation with leptin).

Correlations between appetite regulation (TFEQ) and BOLD signal changes as a result of olanzapine treatment. The most interesting correlations were those observed between changes in appetite regulation and those in BOLD response to appetitive stimuli following olanzapine treatment. Here we found only positive and significant correlations between TFEQ Factor 2 (disinhibition) and changes in BOLD signal in both fusiform gyri $(r=+0.498$; $P<0.05, \quad N=15$ and $r=+0.449 ; P<0.05, N=15)$ and amygdala $(r=+0.449 ; \quad P<0.05, \quad N=15)$. These correlations indicate that perturbations in appetite regulation, especially the disinhibition in the face of food-related stimuli, are associated with an increased neuronal response to these stimuli in the limbic system.

Brain regions sensitive to appetitive stimuli in schizophrenic patients under olanzapine relative to healthy controls. When comparing the group differences for the contrast $A C>N C$, we observed a wide network that was more activated among patients than among healthy controls (Table 4). This network was not only similar to that observed in the previous study, where patients were under various antipsychotic medications, except olanzapine, but this time, the areas were much larger (for example, bigger volume at the same threshold), in line with the increase in appetite dysregulation after the onset of the treatment.

\section{Discussion}

In this study, we investigated the physiological, psychological and neuronal activity changes in schizophrenic patients following 16 weeks of olanzapine treatment. Even after such a short time of taking olanzapine, patients presented important physiological changes, including weight gain, which correlated with changes in the concentration of some metabolites. The negative relationship between body weight and ghrelin plasma concentration found in this study is well established in the literature on obesity ${ }^{29,30}$ and lower ghrelin levels are also found in some eating disorders, such as binge eating. ${ }^{31}$ Similarly, increased levels of triglycerides, such as that found in our results, have been associated in the past with overeating in rats, ${ }^{32}$ and with bulimia nervosa and binge eating in humans. ${ }^{33,34}$ Although leptin is an appetite-reducing hormone, higher levels of leptin for longer periods of time may, in fact, indicate appetite dysfunction due to the development of resistance to leptin, such as it is the case in obese individuals. $^{35}$ In addition, there is a strong association between higher levels of leptin and insulin resistance in both normoglycemic individuals ${ }^{36}$ and type 2 diabetes. ${ }^{37}$ In summary, these physiological changes suggest that hormonal balance involved in appetite regulation may be perturbed in schizophrenic patients after 16 weeks of olanzapine treatment, suggesting that these individuals may be at risk of developing metabolic and/or cardiovascular disorders as a direct consequence of medication. ${ }^{17}$ In fact, the recommendations outlined by De Hert and co-workers ${ }^{17}$ to screen and monitor schizophrenic patients taking atypical antipsychotics are more important in the light of our results: we were able to detect these changes even after a relatively short interval of taking the medication.

We also found activation in the frontal cortex, fusiform gyrus, amygdala and insula when comparing the brain activity in response to appetite-related stimuli after the treatment relative to baseline. It is interesting to note that some of these latter brain regions are part of the limbic system, typically involved in the processing of appetite regulation information. In our experiment, we found hyperactivity in these regions relative to controls, which is the opposite of what is typically found in tasks that required cognitive processing of food images in anorexia nervosa, for example. ${ }^{38}$ However, these findings are in concordance with a new perspective of eating as an automatic behavior ${ }^{39}$ and the evidence that schizophrenic individuals are more sensitive to external cues, ${ }^{40}$ which in our case increased after the olanzapine treatment. The novelty of our findings is that although antipsychotic treatment brought the activity in fusiform gyrus to levels comparable with that of healthy individuals, its functional role in behavioral control of appetite in response to metabolic perturbations ${ }^{41,42}$ seems compromised.

Parsing the salient variables in people with mental illness such as schizophrenia is a crucial need as mortality from physical health causes is the most common cause of premature mortality in people with chronic mental illness. ${ }^{43}$

Table 4 Brain regions in which the difference in BOLD signal between AC and NC is significantly higher among patients than among healthy controls after the olanzapine treatment

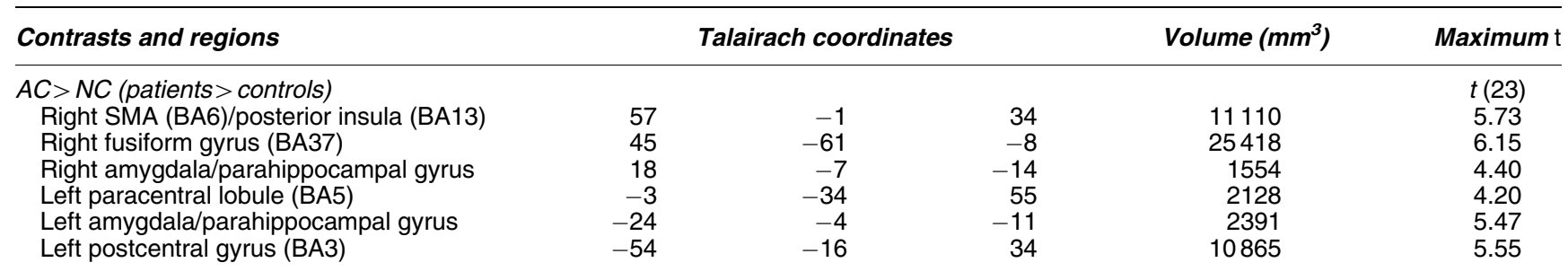

Abbreviations: AC, appetitive condition; BA, Brodmann area; BOLD, blood oxygenation level dependent; NC, neutral condition; SMA, supplementary motor area. The $t$-value represents the magnitude of the increase in the difference between conditions $(A C>N C)$ from pre- to post-treatment. The $t$-value represents the magnitude of the difference between the two groups when considering the difference between conditions $(A C>N C)$. 
Recent molecular examinations have assumed biological differences in hunger, satiation and satiety mechanisms in schizophrenia and have focused on metabolic signals. ${ }^{40,44}$ Here we found that these signals, which were perturbed by olanzapine treatment, correlated both with changes in neuronal activity in the fusiform gyrus and limbic system (amygdala and insula) during the presentation of appetitive images, as well as with measurements indicating a dysfunction in cognitive control of appetite.

Examining differences in neural responses to food pictures between, for instance, obese and lean individuals may help us understand weight-related differences in responses to 'reallife' external cues, such as food displays. In fMRI studies, using a block design, Rothemund et al. ${ }^{45}$ and Stoekel et al. ${ }^{46}$ assessed responses to images of high calorie foods, and low and neutral images following abstinence. The obese individuals showed greater activation to high calorie foods vs neutral in the insula and caudate/putamen. ${ }^{45,46}$

These trials suggest that, similar to our sample olanzapinetreated patients who are more vulnerable to become overweight, obesity is associated with abnormal responses to visual food cues in a distributed network of brain regions that might be involved in reward/motivation, emotion/memory and, more recently, to proximal salience. ${ }^{47}$ Although it is difficult to infer cognitive functions from brain activation, this could reflect individuals' association of food cues with cognitive efforts to restrain intake. ${ }^{22}$ The insula is proposed to play a specific role in a salient network, which, according to Planiyappan and Liddle, ${ }^{47}$ could be a mechanism of dysfunctional psychosis. It is possible that greater reward response in restrainers may be related to a phenotype that comes with restraint: disinhibition, that is, the tendency for restraint to break down when confronted by emotional or external cues to eat. ${ }^{48}$ In DelParigi et al.'s ${ }^{49}$ positron emission topmography study, obese adults who showed greater post-fast increases in activation to food tastes in the midbrain and insula also reported higher disinhibition.

Theoretically, an important candidate mechanism for olanzapine and other second-generation antipsychotics (SGA)-induced increases in appetite is the blockade of receptors such as 5-HT2A, 5-HT2C serotonergic; D1, D2, D3 and D4 dopaminergic; $\mathrm{H} 1$ histaminergic, $\alpha 1-$ and $\alpha 2-$ adrenergic and cholinergic ${ }^{50-58}$ involved in appetite regulation. Some clinical trials implicate the polymorphism of the 5$\mathrm{HT} 2 \mathrm{C}$ receptor gene in weight gain in response to SGAs. ${ }^{58-61}$ Wirshing and co-workers ${ }^{53}$ found a logarithmic relationship between $\mathrm{H} 1$ receptor affinity in patients treated with clozapine (N1/420), olanzapine (N1/413), risperidone (N1/438), haloperidol (N1/443) and sertindole (N1/48), but this is not specific to SGAs. ${ }^{62}$ Nevertheless, rank ordering of specific SGAs' propensities to increase body weight against all their binding affinities at the human brain receptors argues against antagonism of a single receptor as a sufficient cause for SGA-induced weight gain. ${ }^{55,56}$ For instance, ziprasidone also binds with substantial affinity to the 5-HT2C and $\mathrm{H}_{1}$ receptors ${ }^{63}$ Hence, it is possible that simultaneous blockade of several (rather than single) receptors, for example, $\mathrm{H1}, 5$ HT2, D2 and acetylcholine receptors, may have a synergistic effect on increased appetite and food intake..$^{5,64,65}$ It might be that the muscarinic receptor M3 is involved as antipsychotics, which do not produce metabolic side effects, have no affinity for this receptor. However, what seems more relevant from a clinical perspective is that changes in dopaminergic brain pathways induced by long-term treatment with olanzapine are accompanied by metabolic changes and perturbations in the behavioral control of appetite, which are similar to those observed on short term in healthy subjects injected intravenously with ghrelin. ${ }^{42}$

The majority of antipsychotics have been tested to measure if they modify the cognitive mechanism of schizophrenia anomalies. In the obesity or appetite research field, some studies are attempting to break down the mechanisms underlying excessive food intake by moving beyond food intake and food cue paradigms into the domain of cognitive tasks designed to test impulsive behavior such as the go/nogo task ${ }^{66}$ or tasks designed to tap responsiveness to different types of reward. ${ }^{67}$ Our study design is a first step in combining a cognitive paradigm (here implicit) to a food-related information processing.

\section{Limitations}

Given that the patients are on medication, it is therefore difficult to draw conclusions on the processes underlying the performance and activation per se in schizophrenia. This question can only be answered while examining medicationnaïve patients. Evaluating patients before introducing treatment is one way to dissect the effect of schizophrenia per se from the impact of treatment. We also did not employ the best control condition, such as another treatment arm with a weight neutral antipsychotic drug. One limitation of this study is that the control group was only scanned once, resulting in noise. It might be possible that there was a test-retest effect in the schizophrenic sample (for example, difference in familiarity with the paradigm). If the controls had been re-scanned as well, we would have been able to control some of these testretest effects. However, given that our participants did not have to process cognitively the images that were presented, but only to watch and react to them emotionally, there is evidence coming from past studies, which suggests that the emotional response to the same IAPS images tends to be stable over time. ${ }^{68}$ In addition, the comparisons of interest are the changes in physiological, psychological and neuroimaging variables due to the 16 weeks of treatment, seen in patients. We thus used the healthy controls just to assess whether the treatment leads, in patients, to restoration of the brain activity to levels comparable to that of controls. Also, our current design might not be sufficient to distinguish nonspecific changes in responsiveness from specific cognitive changes related to the appetite-related cognitive processes that they are interested in. The time patients stay in the MRI set up was long enough for them and we did not add other paradigms.

\section{Conclusion}

The epidemic of obesity is probably related to the multiple 'obesogenic' influences in modern environment. However, despite the unfortunate attraction of fast food restaurants and large portion sizes, not everyone becomes obese, suggesting 
that individuals such as patients with schizophrenia differ in their susceptibility to environmental opportunities to eat. This vulnerability can also be magnified by the medication they take.

In this study, we documented that schizophrenic patients undergoing a treatment with olanzapine are likely to gain weight, augment their abdominal fat matter and increase their levels of insulin and leptin, while diminishing the ghrelin blood concentration. Interestingly, these physiological changes following the olanzapine treatment were paralleled by an increased level of activity in the limbic system (amygdala, insula) in patients relative to controls. Moreover, the neuronal activity in the amygdala and insula in response to appetitive stimuli correlated with several physiological measurements related to appetite regulation. The changes in insulin, leptin and ghrelin concentrations in our study and the increase in neuronal activity of the amygdala, which is the site typically integrating homeostatic and extrinsic influences related to eating behavior, might be consistent with the hypothesis of a dopaminergic-mediated feeding control. However, this question remains open as it is still difficult to determine precisely why this dopamine mediation would be different with olanzapine as compared to some of the other antipsychotics, including the first generation. One possible explanation could be that this is related to the mechanisms of neurotransmitter mediation. The only study dealing with this mechanism is by Albaugh and co-workers, ${ }^{18}$ who showed in rats that olanzapine led to specific metabolic effects that increased the accumulation of fuel into the adipose tissue. The clinical significance of our study stems from the fact that, although the investigated duration of the treatment was relatively short (about 4 month), it was enough to reveal changes in the brain activity and in other physiological parameters that reflect changes in appetite regulation induced by antipsychotic medication. Our study, while still exploratory, suggests that olanzapine treatment in humans induced changes in neuronal activity in specific brain regions, which correlate with levels of glucose, insulin, prolactin or the cholesterol/LDL ratio. Further research is needed to parse the pharmacological and neuronal mechanisms producing the metabolic side effects during antipsychotic treatment in schizophrenia.

\section{Conflict of interest}

The authors declare no conflict of interest.

Acknowledgements. This study was conducted as part of investigator initiated trials (No: clinical trial: NCT 00290121) funded by Eli Lilly Canada.

1. De Hert M, Vancampfort D, Correll CU, Mercken V, Peuskens J, Sweers K et al. Guidelines for screening and monitoring of cardiometabolic risk in schizophrenia: systematic evaluation. Br J Psychiatry 2011; 199: 99-105.

2. Rummel-Kluge $\mathrm{C}$, Komossa $\mathrm{K}$, Schwarz $\mathrm{S}$, Hunger $\mathrm{H}$, Schmid F, Lobos $\mathrm{CA}$ et al. Head-tohead comparisons of metabolic side effects of second generation antipsychotics in the treatment of schizophrenia: a systematic review and meta-analysis. Schizophr Res 2010; 123: $225-233$.

3. Thornton-Jones Z, Neill JC, Reynolds GP. The atypical antipsychotic olanzapine enhances ingestive behaviour in the rat: a preliminary study. J Psychopharmacol (Oxford, England) 2002; 16: 35-37.
4. Ota M, Mori K, Nakashima A, Kaneko YS, Fujiwara K, Itoh M et al. Peripheral injection of risperidone, an atypical antipsychotic, alters the bodyweight gain of rats. Clin Exp Pharmacol Physiol 2002; 29: 980-989.

5. Hartfield AW, Moore NA, Clifton PG. Effects of clozapine, olanzapine and haloperidol on the microstructure of ingestive behaviour in the rat. Psychopharmacology 2003; 167: 115-122.

6. Arjona AA, Zhang SX, Adamson B, Wurtman RJ. An animal model of antipsychoticinduced weight gain. Behav Brain Res 2004; 152: 121-127.

7. Cope MB, Nagy TR, Fernandez JR, Geary N, Casey DE, Allison DB. Antipsychotic druginduced weight gain: development of an animal model. Int J Obes 2005; 29: 607-614.

8. Bromel T, Blum WF, Ziegler A, Schulz E, Bender M. Fleischhaker C et al. Serum leptin levels increase rapidly after initiation of clozapine therapy. Mol Psychiatry 1998; 3: 76-80.

9. Briffa D, Meehan T. Weight changes during clozapine treatment. Aust N Z J Psychiatry 1998; 32: 718-721.

10. Eder U, Mangweth B, Ebenbichler C, Weiss E, Hofer A, Hummer M et al. Association of olanzapine-induced weight gain with an increase in body fat. Am J Psychiatry 2001; 158: 1719-1722.

11. Basson BR, Kinon BJ, Taylor CC, Szymanski KA, Gilmore JA, Tollefson GD. Factors influencing acute weight change in patients with schizophrenia treated with olanzapine, haloperidol, or risperidone. J Clin Psychiatry 2001; 62: 231-238.

12. Kinon BJ, Kaiser CJ, Ahmed S, Rotelli MD, Kollack-Walker S. Association between early and rapid weight gain and change in weight over one year of olanzapine therapy in patients with schizophrenia and related disorders. J Clin Psychopharmacol 2005; 25: 255-258.

13. Gothelf D, Falk B, Singer P, Kairi M, Phillip M, Zigel $L$ et al. Weight gain associated with increased food intake and low habitual activity levels in male adolescent schizophrenic inpatients treated with olanzapine. Am J Psychiatry 2002; 159: 1055-1057.

14. Kane JM, Barrett EJ, Casey DE, Correll CU, Gelenberg AJ, Klein S et al. Metabolic effects of treatment with atypical antipsychotics. J Clin Psychiatry 2004; 65: 1447-1455.

15. Lublin H, Eberhard J, Levander S. Current therapy issues and unmet clinical needs in the treatment of schizophrenia: a review of the new generation antipsychotics. Int Clin Psychopharmacol 2005; 20: 183-198.

16. Theisen FM, Linden A, Konig IR, Martin M, Remschmidt H, Hebebrand J. Spectrum of binge eating symptomatology in patients treated with clozapine and olanzapine. J Neural Transm 2003; 110: 111-121.

17. De Hert M, Detraux J, van Winkel R, Yu W, Correll CU. Metabolic and cardiovascular adverse effects associated with antipsychotic drugs. Nat Rev Endocrinol 2011; 8: 114-126.

18. Albaugh VL, Judson JG, She P, Lang CH, Maresca KP, Joyal JL et al. Olanzapine promotes fat accumulation in male rats by decreasing physical activity, repartitioning energy and increasing adipose tissue lipogenesis while impairing lipolysis. Mol Psychiatry 2011; 16: 569-581.

19. $\mathrm{Ng} \mathrm{J}$, Stice E, Yokum S, Bohon C. An fMRI study of obesity, food reward, and perceived caloric density. Does a low-fat label make food less appealing? Appetite 2011; 57: 65-72.

20. Burger KS, Stice E. Relation of dietary restraint scores to activation of reward-related brain regions in response to food intake, anticipated intake, and food pictures. Neurolmage 2011; 55: 233-239.

21. Stice E, Spoor S, Bohon C, Veldhuizen MG, Small DM. Relation of reward from food intake and anticipated food intake to obesity: a functional magnetic resonance imaging study. $J$ Abn Psychol 2008; 117: 924-935.

22. Lungu O, Anselmo K, Letourneau G, Mendrek A, Stip B, Lipp O et al. Neuronal correlates of appetite regulation in patients with schizophrenia: Is there a basis for future appetite dysfunction? Eur Psychiatry; advance online publication, 26 April 2012 (e-pub ahead of print)

23. Lang PJ, Öhman A, Vaitl D. The International Affective Picture System (photographic slides). Center for Research in Psychophysiology, University of Florida: Gainesville, FL, 1988

24. Kay SR, Fiszbein A, Opler LA. The positive and negative syndrome scale (PANSS) for schizophrenia. Schizophr Bull 1987; 13: 261-276.

25. Addington D, Addington J, Schissel B. A depression rating scale for schizophrenics. Schizophr Res 1990; 3: 247-251.

26. Stunkard AJ, Messick S. The three-factor eating questionnaire to measure dietary restraint, disinhibition and hunger. J Psychosomat Res 1985; 29: 71-83.

27. Talairach J, Tournoux P. Co-Planar Stereotaxic Atlas of the Human Brain. Thieme Medical Publishers: New York, 1988

28. Penny WD, Holmes AJ. Random-effects analysis. In: Frackowiak RSJ, Friston KJ, Frith C, Dolan R, Friston KJ, Price CJ et al. (eds), Human Brain Function, 2nd edn. Academic Press: London, 2003

29. Kelishadi R, Hashemipour M, Mohammadifard N, Alikhassy H, Adeli K. Short- and longterm relationships of serum ghrelin with changes in body composition and the metabolic syndrome in prepubescent obese children following two different weight loss programmes. Clin Endocrinol 2008; 69: 721-729.

30. Stylianou C, Galli-Tsinopoulou A, Farmakiotis D, Rousso I, Karamouzis M, Koliakos G et al. Ghrelin and leptin levels in obese adolescents. Relationship with body fat and insulin resistance. Hormones (Athens, Greece) 2007; 6: 295-303.

31. Geliebter A, Gluck ME, Hashim SA. Plasma ghrelin concentrations are lower in bingeeating disorder. J Nutr 2005; 135: 1326-1330. 
32. Karatayev O, Gaysinskaya V, Chang GQ, Leibowitz SF. Circulating triglycerides after a high-fat meal: predictor of increased caloric intake, orexigenic peptide expression, and dietary obesity. Brain Res 2009; 1298: 111-122.

33. Monteleone P, Fabrazzo M, Martiadis V, Fuschino A, Serritella C, Milici N et al. Opposite changes in circulating adiponectin in women with bulimia nervosa or binge eating disorder $J$ Clin Endocrinol Metab 2003; 88: 5387-5391.

34. Monteleone P, Santonastaso P, Pannuto M, Favaro A, Caregaro L, Castaldo E et al. Enhanced serum cholesterol and triglyceride levels in bulimia nervosa: relationships to psychiatric comorbidity, psychopathology and hormonal variables. Psychiatry Res 2005; 134: 267-273.

35. Quilliot $D$, Bohme $P$, Zannad F, Ziegler O. Sympathetic-leptin relationship in obesity: effect of weight loss. Metab Clin Exp 2008; 57: 555-562.

36. Haffner SM, Miettinen H, Mykkanen L, Karhapaa P, Rainwater DL, Laakso M. Leptin concentrations and insulin sensitivity in normoglycemic men. Int J Obes Relat Metab Disord 1997; 21: 393-399.

37. Wauters M, Considine RV, Yudkin JS, Peiffer F, De Leeuw I, Van Gaal LF. Leptin levels in type 2 diabetes: associations with measures of insulin resistance and insulin secretion. Horm Metab Res 2003; 35: 92-96.

38. van Kuyck K, Gerard N, Van Laere K, Casteels C, Pieters G, Gabriels L et al. Towards a neurocircuitry in anorexia nervosa: evidence from functional neuroimaging studies. $J$ Psychiatric Res 2009; 43: 1133-1145.

39. Cohen D, Farley TA. Eating as an automatic behavior. Prev Chronic Dis 2008; 5: A23.

40. Sentissi O, Viala A, Bourdel MC, Kaminski F, Bellisle F, Olie JP et al. Impact of antipsychotic treatments on the motivation to eat: preliminary results in 153 schizophrenic patients. Int Clin Psychopharmacol 2009; 24: 257-264.

41. Dagher A. The neurobiology of appetite: hunger as addiction. Int J Obes 2009; 33(Suppl 2): S30-S33.

42. Malik S, McGlone F, Bedrossian D, Dagher A. Ghrelin modulates brain activity in areas that control appetitive behavior. Cell Metab 2008; 7: 400-409.

43. Taylor VH, Mclntyre RS, Remington G, Levitan RD, Stonehocker B, Sharma AM. Beyond pharmacotherapy: understanding the links between obesity and chronic mental illness. Can J Psychiatry 2012; 57: 5-12.

44. Schanze A, Reulbach U, Scheuchenzuber M, Groschl M, Kornhuber J, Kraus T. Ghrelin and eating disturbances in psychiatric disorders. Neuropsychobiology 2008; 57: 126-130.

45. Rothemund $\mathrm{Y}$, Preuschhof $\mathrm{C}$, Bohner G, Bauknecht $\mathrm{HC}$, Klingebiel R, Flor $\mathrm{H}$ et al. Differential activation of the dorsal striatum by high-calorie visual food stimuli in obese individuals. Neurolmage (Research Support, Non-US Gov't) 2007; 37: 410-421.

46. Stoeckel LE, Weller RE, Cook EW, Twieg DB, Knowlton RC, Cox JE. Widespread rewardsystem activation in obese women in response to pictures of high-calorie foods. Neurolmage 2008; 41: 636-647.

47. Palaniyappan L, Liddle PF. Does the salience network play a cardinal role in psychosis? An emerging hypothesis of insular dysfunction. J Psychiatr Neurosci 2012; 37: 17-27.

48. Herman CP. Restrained eating. In: Stunkard A (ed.) Obesity. WB Saunders: Philadelphia, PA, 1980, pp 208-225

49. DelParigi A, Chen KW, Salbe AD, Reiman EM, Tataranni PA. Sensory experience of food and obesity: a positron emission tomography study of the brain regions affected by tasting a liquid meal after a prolonged fast. Neurolmage 2005; 24: 436-443.

50. Seeman P, Tallerico T, Corbett R, Van Tol HH, Kamboj RK. Role of dopamine D2, D4 and serotonin(2A) receptors in antipsychotic and anticataleptic action. $J$ Psychopharmacol (Oxford, England) 1997; 11: 15-17.
51. Van Tol HH, Bunzow JR, Guan HC, Sunahara RK, Seeman P, Niznik HB et al. Cloning of the gene for a human dopamine D4 receptor with high affinity for the antipsychotic clozapine. Nature 1991; 350: 610-614.

52. Zeng XP, Le F, Richelson E. Muscarinic $m 4$ receptor activation by some atypical antipsychotic drugs. Eur J Pharmacol 1997; 321: 349-354.

53. Wirshing DA, Marshall Jr BD, Green MF, Mintz J, Marder SR, Wirshing WC. Risperidone in treatment-refractory schizophrenia. Am J Psychiatry 1999; 156: 1374-1379.

54. Meltzer HY. The role of serotonin in antipsychotic drug action. Risperidone in treatment-refractory schizophrenia. Neuropsychopharmacology 1999; 21(Suppl): 106S-115S.

55. Richelson E. Receptor pharmacology of neuroleptics: relation to clinical effects. J Clin Psychiatry 1999; 60(Suppl 10): 5-14.

56. Richelson E, Souder T. Binding of antipsychotic drugs to human brain receptors focus on newer generation compounds. Life Sci 2000; 68: 29-39.

57. Casey DE, Zorn SH. The pharmacology of weight gain with antipsychotics. J Clin Psychiatry 2001; 62(Suppl 7): 4-10.

58. Reynolds GP, Templeman LA, Zhang ZJ. The role of $5-\mathrm{HT} 2 \mathrm{C}$ receptor polymorphisms in the pharmacogenetics of antipsychotic drug treatment. Progr Neuro-Psychopharmacol Biol Psychiatry 2005; 29: 1021-1028.

59. Tsai SJ, Hong CJ, Yu YW, Lin $\mathrm{CH}$. $-759 \mathrm{C} / \mathrm{T}$ genetic variation of $5 \mathrm{HT}(2 \mathrm{C})$ receptor and clozapine-induced weight gain. Lancet 2002; 360: 1790.

60. Basile VS, Masellis M, De Luca V, Meltzer HY, Kennedy JL. $759 \mathrm{C} / \mathrm{T}$ genetic variation of $5 \mathrm{HT}(2 \mathrm{C})$ receptor and clozapine-induced weight gain. Lancet 2002; 360: 1790-1791.

61. Ellingrod VL, Perry PJ, Ringold JC, Lund BC, Bever-Stille K, Fleming F et al. Weight gain associated with the $-759 \mathrm{C} / \mathrm{T}$ polymorphism of the $5 \mathrm{HT} 2 \mathrm{C}$ receptor and olanzapine. Am J Med Genet B 2005; 134B: 76-78.

62. Allison DB, Mentore JL, Heo M, Chandler LP, Cappelleri JC, Infante MC et al. Antipsychotic-induced weight gain: a comprehensive research synthesis. Am J Psychiatry 1999; 156: 1686-1696.

63. Pouzet B, Mow T, Kreilgaard M, Velschow S. Chronic treatment with antipsychotics in rats as a model for antipsychotic-induced weight gain in human. Pharmacol Biochem Behav 2003; 75: 133-140.

64. Masand PS, Gupta S. Selective serotonin-reuptake inhibitors: an update. Harvard Rev Psychiatry 1999; 7: 69-84.

65. Wetterling T. Bodyweight gain with atypical antipsychotics. A comparative review. Drug Saf 2001; 24: 59-73.

66. Batterink L, Yokum S, Stice E. Body mass correlates inversely with inhibitory control in response to food among adolescent girls: an fMRI study. Neurolmage 2010; 52 : 1696-1703.

67. Stice E, Yokum S, Burger KS, Epstein LH, Small DM. Youth at risk for obesity show greater activation of striatal and somatosensory regions to food. J Neurosci 2011; 31: 4360-4366.

68. Broome R, Gard DE, Mikels JA. Test-retest reliability of an emotion maintenance task Cogn Emot 2012; 26: 737-747.

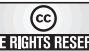

Translational Psychiatry is an open-access journal published by Nature Publishing Group. This work is licensed under the Creative Commons Attribution-Noncommercial-Share Alike 3.0 Unported License. To view a copy of this license, visit http:// creativecommons.org/licenses/by-nc-sa/3.0/ 